for dLC), the difference in net monetary benefit was around $£ 700$ per patient in favour eLC pathway. In a hospital conducting around 200 cholecystectomies per year on patients for acute biliary disease in the NHS, this could translate to a cost saving of over $£ 140000$ per year.

Competing interests None declared.

\section{OC-131 PATTERN OF RECURRENCE FOLLOWING NON- ANATOMICAL RESECTION FOR COLORECTAL LIVER METASTASIS}

doi:10.1136/gutjnl-2012-302514a.131

A Hakeem,* G Marangoni, A Nair, R Young, E Hidalgo, G Toogood, J P A Lodge, K R Prasad. Department of HPB Surgery, St James's University Hospital NHS Trust, Leeds, UK

Introduction Non-anatomical resection (NAR) for colorectal liver metastasis (CRLM) has become more common in an attempt to preserve liver parenchyma. Pattern of recurrence (POR) following NAR has been reported previously, but there has been no detail on the hepatic recurrences with respect to previous NAR. We aim to investigate pattern of hepatic recurrence in patients who underwent NAR for CRLM, especially looking at the recurrences in the context of previous resected segment.

Methods 1580 CRLM resection were performed in our centre between 1993 and 2010. 591 (37.4\%) were NAR and 989 (62.6\%) were AR. Patients who underwent simultaneous Anatomical Resection (AR) were excluded (95 out of 591). All NAR were grouped as either unilobar or bilobar metastatectomy and POR studied in relation to previous resected segment.

Results 495 NAR were included. There were 291 recurrences (58.7\%). 188 were hepatic (37.9\%) and 103 extra-hepatic (20.8\%). In 11 patients with hepatic recurrence, data were not available for recurrence segment and therefore excluded from POR analysis. The POR following unilobar metastatectomy ( $n=127)$ : same segment ( $n=32,25.2 \%)$, same sector $(n=9,7.1 \%)$, ipsilateral hemiliver $(n=48,37.8 \%)$, contralateral hemi-liver $(n=15,11.8 \%)$ and bilobar ( $\mathrm{n}=23,18.1 \%)$. The POR following bilobar metastatectomy $(n=50)$ : same segment $(n=1,2 \%)$, hemi-liver with equal number of segments resected previously $(n=15,30 \%)$, hemi-liver with lesser involved segments previously $(n=7,14 \%)$, hemi-liver with more segment involved previously $(n=10,20.0 \%)$ and bilobar $(n=17$, $34.0 \%$ ).

Conclusion This retrospective study shows that following unilobar CRLM resections, $70.0 \%$ recur in the ipsilateral hemi-liver. In bilobar resections, $22.0 \%$ recur in the same segment or hemi-liver with previous multiple resections. Our data supports a more aggressive approach for unilobar disease. Further studies need to be done on pattern of recurrences in patients undergoing parenchyma preserving surgery to develop best surgical strategies.

Competing interests None declared.

\section{OC-132 CARDIOPULMONARY EXERCISE TESTING INCREASES HEPATECTOMY RATE IN THE ELDERLY}

doi:10.1136/gutjnl-2012-302514a.132

${ }^{1} \mathrm{D}$ Dunne, ${ }^{*} \mathrm{R}$ Jones, ${ }^{1} \mathrm{H}$ Malik, ${ }^{1} \mathrm{G}$ Poston, ${ }^{2} \mathrm{D}$ Palmer, ${ }^{3} \mathrm{~S}$ Jack, ${ }^{1} \mathrm{~S}$ Fenwick. ${ }^{1}$ Northwestern Hepatobiliary Centre, Aintree University Hospital, Liverpool, UK; ${ }^{2}$ Department of Oncology, University of Liverpool, Liverpool, UK; ${ }^{3}$ Department of Respiratory Medicine, Aintree University Hospital, Liverpool, UK

Introduction Hepatectomy offers the only possibility of cure to patients with colorectal liver metastases (CRLM) and although 50\% of CRLM patients are $>70$, only $25 \%$ of those undergoing hepatectomy are $>70 .{ }^{1}$ This is likely to be due to the higher perioperative mortality. Cardiopulmonary exercise testing (CPET) can identify patients at higher operative risk. ${ }^{2}$ We introduced CPET on 1 October 2009. This study assesses its effect on patient selection and outcome.

Methods After 1 October 2009 all patients undergoing resection of CRLM meeting one of the following criteria underwent CPET. Criteria

1. Planned extended right/or extended left resection

2. Over 65

3. Significant comorbidities

Data were collected prospectively. Group (A) 1 October 2009-21 February 2011 was compared to a published historical series 1 August 1990 to 1 April 2007 (Group B). We also compared our results to a recently published series from LiverMetSuvey (LMS). ${ }^{1}$

Results Group A 155 patients, Group B 654 patients. In Group A 64 (41\%) patients were 70+, Group B $181(28 \%)$ patients $(\mathrm{p}<0.0001)$. Within the over 70's groups here was no difference in male:female ratio or postoperative complications, but there was an increased use of neoadjuvant chemotherapy in Group A 26 patients (40\%) vs 34 patients $(19 \%)$ Group B ( $p=0.001)$. In hospital mortality was $1.6 \%$ (1 patient) Group A vs 4.9\% ( 9 patients) group B ( $p=0.46)$. Comparing Group A to LMS data ${ }^{1}$ from 1/1986 to 6/2007 (7764 resections). Group A $41 \%$ aged $70+$ vs $21 \%$ LMS $(p<0.01)$. No significant difference in neoadjuvant chemotherapy use (Group A $41 \%$ vs $\operatorname{LMS} 34 \%)(p=0.22)$, or 60 day mortality (Group $A-1$ patient $(1.6 \%)$ vs $62(3.8 \%)$ LMS) $(p=0.51)$

Conclusion CPET enabled accurate preoperative fitness assessment and led to a significant increase in the number of patients over the age of 70 proceeding to surgery. There is a trend towards lower mortality possibly due to better preoperative assessment. We believe that the number of hepatectomies undertaken for CRLM in patients aged over 70 would rise if CPET were introduced as a standard preoperative assessment tool.

Competing interests None declared.

\section{REFERENCES}

1. Adam R, Frilling A, Elias D, et al; LiverMetSurvey Centres. Liver resection of colorectal metastasis in elderly patients. Br J Surg 2010;97:366-76.

2. Smith TB, Stonell C, Purkayastha $S$, et al. Cardiopulmonary exercise testing as a risk assessment method in non cardio-pulmonary surgery: a systematic review. Anaesthesia 2009;64:883-93.

\section{OC-133 ARTERY FIRST PPPD—CLINICAL OUTCOME AND STAGING USING A STANDARDISED PATHOLOGY PROTOCOL}

doi:10.1136/gutjnl-2012-302514a.133

D J Malde, * G Morris-Stifff, C Verbeke, A M Smith. The HPB Unit, St James University Hospital, Leeds, UK

Introduction With increased numbers of borderline locally advanced patients undergoing resection due to the acceptance of portal vein (PV) resection, early dissection of the superior mesenteric artery (SMA) allows definitive assessment of the tumour/SMA relationship and improved control when PV resection is required.

Methods The lymph node yield, resection margins, morbidity and mortality data were compared between patients undergoing Artery first technique of pancreaticoduodenectomy (January 2009-December 2010) and previous resections (January 2000-December 2008).

Results 42 resections were carried out using the artery first technique were age, sex and pathology matched with 182 previous 